Boletim Técnico da Divisão de Experimentação e Pesquisas INSTITUTO A GRONÔMICO

\begin{tabular}{lll}
\hline \hline Vol. 10 & Campinas, Dezembro de 1950 & N. ${ }^{\circ} 12$ \\
\hline \hline
\end{tabular}

\title{
A SECAGEM DE AMIDO PELO AR QUENTE (1)
}

Andre Tosello, engenheiro agrônomo, Secção de Café, e ARI de Arruda Veiga, engenheiro agrônomo, Seccãas de Tecnologia, Instituto Agronômico do Campinas

\section{1 - INTRODUÇÃO}

As indústrias de amido, no Estado de São Paulo, empregam geralmente a secagem a vácuo. Êste processo é recomendável para se obter um bom produto, porém é moroso e caro. O amido que deve sofrer a operação da secagem é submetido prèviamente à centrifugação, até que passe a ter um teor de umidade da ordem de 30 a $40 \%$.

Tem-se constantemente combatido o emprêgo dos secadores a ar quente para o amido, pelo fato de se provocar a formação de dextrina no produto. Quando é empregado êste tipo de secador, tem-se recomendado trabalhar a uma temperatura máxima de $40^{\circ} \mathrm{C}$. $\hat{\mathrm{E}}$ evidente que esta temperatura torna antieconômico o emprêgo dêste processo.

Os ensaios aqui mencionados foram iniciados em 1947 e repetidos em 1948 e por êles se verifica ser perfeitamente possível o emprêgo dos secadores a ar quente, para o amido, desde que a marcha da secagem seja feita racionalmente.

A temperatura limite de secagem não é um dado fixo para o produto, mas depende principalmente do seu teor de umidade. Dêsse modo, à medida que o produto vai secando, a temperatura limite de secagem deve subir. Num secador baseado no princípio de contra correntes, é, portanto, perfeitamente viável a secagem a temperaturas bastante altas.

No presente trabalho procura-se estabelecer a correlação existente entre os três fatôres, quais sejam : a) teor de umidade do produto ; $b$ ) temperatura de secagem; c) percentagem de dextrina.

\section{2 - MATERIAL, MÉTODO E RESULTADOS OBTIDOS}

De uma só amostra de amido, retirado da centrífuga com cêrca de $30 \%$ de umidade, foram tomadas 72 porções de 50 gramas cada uma e submetidas a secagens parciais à baixa temperatura $\left(30^{\circ} \mathrm{C}\right)$ em estufa. Cada

(1) Trabalho apresentado no 8. ${ }^{\circ}$ Congresso Internacional de Indústrias Agrícolas, realizado, em Bruxelas, em julho de 1950 . 
grupo de 8 porções foi retirado, num total de 9 , a intervalos diferentes, a fim de se obterem amostras com diferentes teores de umidade. Dêsse modo foi possível organizar as seguintes amostras :

\begin{tabular}{|c|c|c|c|}
\hline $\begin{array}{cc}\text { NúMERo DA } & \text { Umidade } \\
\text { AMOSTRA } & \%\end{array}$ & $\begin{array}{cc}\text { NGMero DA } & \text { Umidade } \\
\text { AMOSTRA } & \%\end{array}$ & $\begin{array}{l}\text { NÚMERO DA } \\
\text { AMOSTRA }\end{array}$ & $\underset{\%}{U}$ \\
\hline 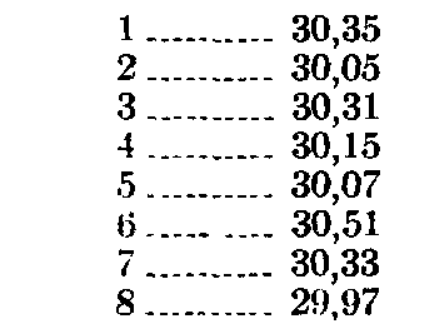 & $\begin{array}{l}25 \ldots \ldots \ldots \\
26 \\
27\end{array} \ldots \ldots$ & $\begin{array}{l}49 \\
50 \\
51 \\
52\end{array}$ & $\begin{array}{l}15,24 \\
15,19 \\
18,01 \\
15,58 \\
16,35 \\
14,86 \\
14,54 \\
14,98\end{array}$ \\
\hline 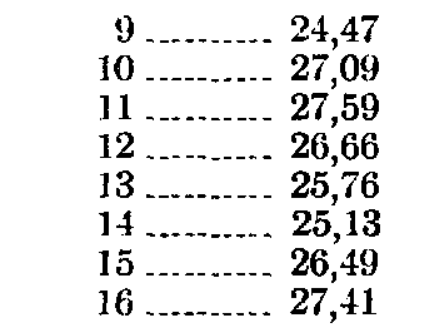 & 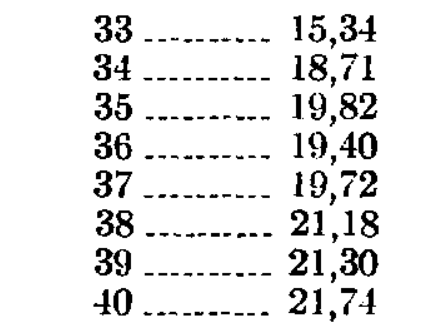 & $\begin{array}{l}5 \overline{6} \\
58 \ldots \ldots \\
59 \\
60 \\
61 \\
62 \\
63 \\
64\end{array}$ & $\begin{array}{l}13,60 \\
13,90 \\
14,24 \\
13,51 \\
12,69 \\
12,74 \\
14,10 \\
13,85\end{array}$ \\
\hline $\begin{array}{l}17 \ldots \ldots \ldots \\
18 \ldots \ldots \ldots \\
19 \\
20 \ldots \ldots \ldots\end{array}$ & 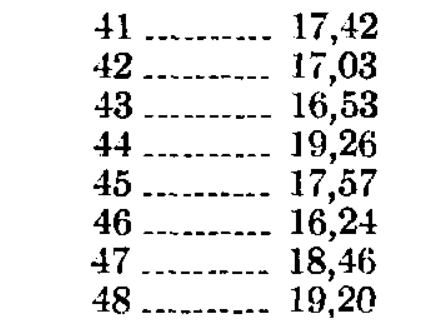 & $\begin{array}{l}65 \\
66 \\
67\end{array} 6 \ldots \ldots \ldots$ & $\begin{array}{r}9,50 \\
-\quad 9,65 \\
-\quad 8,18 \\
-\quad 8,96 \\
-11,07 \\
-11,33 \\
-11,09 \\
10,91\end{array}$ \\
\hline
\end{tabular}

Estas amostras foram reagrupadas em classes contendo 9 amostras cada uma; em cada classe, foram colocadas amostras com os diferentes teores de umidade, e, em seguida, submetidas à secagem pelo ar quente a diferentes temperaturas, como segue:

$\begin{array}{rrrrrrrr}30^{\circ} \mathrm{C} & 40^{\circ} \mathrm{C} & 50^{\circ} \mathrm{C} & 60^{\circ} \mathrm{C} & 70^{\circ} \mathrm{C} & 80^{\circ} \mathrm{C} & 90^{\circ} \mathrm{C} & 100^{\circ} \mathrm{C} \\ 1 & 2 & 3 & 4 & 5 & 6 & 7 & 8 \\ 9 & 10 & 11 & 12 & 13 & 14 & 15 & 16 \\ 17 & 18 & 19 & 20 & 21 & 22 & 23 & 24 \\ 25 & 26 & 27 & 28 & 29 & 30 & 31 & 32 \\ 33 & 34 & 35 & 36 & 37 & 38 & 39 & 40 \\ 41 & 42 & 43 & 44 & 45 & 46 & 47 & 48 \\ 49 & 50 & 51 & 52 & 53 & 54 & 55 & 56 \\ 57 & 58 & 59 & 60 & 61 & 62 & 63 & 64 \\ 65 & 66 & 67 & 68 & 69 & 70 & 71 & 72\end{array}$

Após a secagem em secador de gaveta a ar quente, os lotes foram analisados, com relação ao teor de dextrina, pelo seguinte método, adotado na Secção de Tecnologia Agrícola, do Instituto Agronômico: 
Pesam-se dez gramas de farinha, transpcrtando-as para a garrafa de Sthomann; trata-se com $100 \mathrm{~cm}^{3}$ de álcool a $8 \%$ e agita-se durante uma hora ; filtra-se em Buchner, lavando-se o resíduo com $150 \mathrm{~cm}^{3}$ de álcool da mesma concentração. Seca-se o pó e passa-se novamente para a mesma garrafa de Sthomann, que também foi submetida à secagem na estufa; juntam-se exatamente $100 \mathrm{~cm}^{3}$ de água, agita-se novamente durante uma hora e adiciona-se uma pitada de $\mathrm{CaO}$ e filtra-se. Transfere-se com uma pipeta $150 \mathrm{~cm}^{3}$ do filtrado, transportando-os a um balão de $100 \mathrm{~cm}^{3}$; juntam-se umas gotas de $\mathrm{HCl}$ diluído, usando-se como indicador o papel de congo, para neutralizar o $\mathrm{CaO}$, e juntam-se depois $10 \mathrm{~cm}^{3}$ de $\mathrm{HCl}$ a $1: 125$ e inverte-se a goma e dextrina durante duas horas em BM em ebulição. Retira-se depois o balão, resfria-se, neutraliza-se com soda, completa-se o volume de $100 \mathrm{~cm}^{3}$ e dosa-se o açúcar segundo Lane ou Bertrand.

Os açúcares redutores foram dosados, pelo método Bertrand, obtendo-se os resultados constantes do quadro 1 .

Qradro 1.-Formação de dextrina no amido, durante o processo de secagem, partindo de amostias com variáveis percentagens de umidade e submetidas a diferentes temperaturas

\begin{tabular}{|c|c|c|c|c|c|c|c|}
\hline $\begin{array}{l}\text { Temperatura } \\
\text { de secagem }\end{array}$ & $\begin{array}{c}\text { Número } \\
\text { da } \\
\text { amostra }\end{array}$ & $\begin{array}{l}\text { Percentagem } \\
\text { de umidade } \\
\text { antes da } \\
\text { secagem }\end{array}$ & Dextrina & $\begin{array}{l}\text { Temperatura } \\
\text { de secagem }\end{array}$ & $\begin{array}{l}\text { Número } \\
\text { da } \\
\text { amostra }\end{array}$ & $\begin{array}{l}\text { Percentagen } \\
\text { de unidade } \\
\text { antes da } \\
\text { secagem }\end{array}$ & Dextrina \\
\hline $30^{\circ} \mathrm{C}$. & 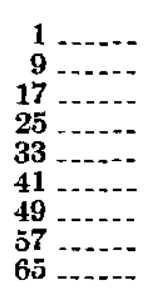 & 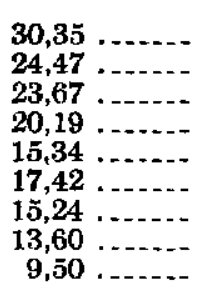 & $\begin{array}{l}0,330 \\
0,140 \\
0,140 \\
0,090 \\
0,090 \\
0,068 \\
0,068 \\
0,068 \\
0,023\end{array}$ & $70^{\circ} \mathrm{C}$. & $\begin{array}{r}5 \\
53 \\
13 \\
21 \\
29 \\
37-\cdots \\
37-\cdots \\
45 \\
53 \\
61 \\
69 \\
69-\cdots \\
\cdots-\cdots \\
\end{array}$ & $\begin{array}{l}30,07 \\
25,76 \\
24,94 \\
20,82 \\
19,72 \\
17,57 \\
16,36 \\
12,69 \\
11,07 \\
\cdots\end{array}$ & $\begin{array}{l}0,900 \\
0,390 \\
0,330 \\
0,230 \\
0,140 \\
0,068 \\
\mathbf{0 , 0 4 5} \\
0,023 \\
\mathbf{0 , 0 6 8}\end{array}$ \\
\hline $40^{\circ} \mathrm{C} \ldots$ & 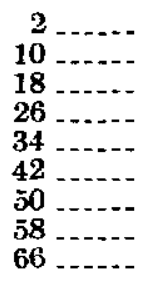 & 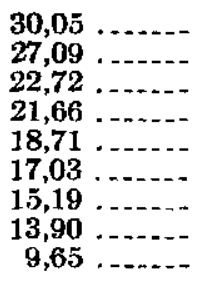 & $\begin{array}{l}0,450 \\
0,440 \\
0,160 \\
0,140 \\
0,090 \\
0,090 \\
0,068 \\
0,068 \\
0,023\end{array}$ & $80^{\circ} \mathrm{C}$ & $\begin{array}{r}6 \\
6 \\
14 \\
22 \\
30 \\
38 \\
38 \\
46 \\
54 \\
52 \\
62 \\
70 \\
\cdots\end{array}$ & $\begin{array}{l}30,51 \\
25,13 \\
23,89\end{array} \ldots$ & \begin{tabular}{l}
1,400 \\
0,500 \\
0,390 \\
0,230 \\
\hdashline 0,068 \\
0,045 \\
0,045 \\
0,068
\end{tabular} \\
\hline $500 \mathrm{C}:-$ & 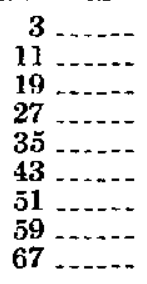 & $\begin{array}{r}30,31, \ldots \\
27,59 \\
21,26 \\
21,90 \\
19,82 \\
16,53 \\
18,01 \\
14,24 \\
8,18 \\
8, \ldots \\
.\end{array}$ & $\begin{array}{l}0,500 \\
0,330 \\
0,160 \\
0,160 \\
0,120 \\
0,090 \\
0,090 \\
0,045 \\
0,068\end{array}$ & $90^{\circ} \mathrm{C} \ldots$ & $\begin{array}{r}7 \\
15 \\
23 \\
31 \\
31 \\
39 \\
47 \\
47 \\
55 \\
63 \\
71 \\
71\end{array}$ & $\begin{array}{l}30,33 \\
26,49 \\
21,90 \\
21,59 \\
21,30 \\
18,4 . \\
14, \ldots \\
14, \ldots \\
14,10 \\
11,09\end{array}$ & $\begin{array}{l}1,600 \\
0,680 \\
0,330 \\
0,330 \\
0,280 \\
0,140 \\
0,068 \\
0,045 \\
0,068\end{array}$ \\
\hline $60^{\circ} \mathrm{C} \ldots$ & 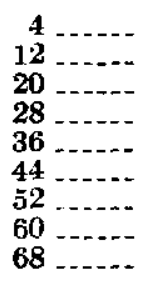 & 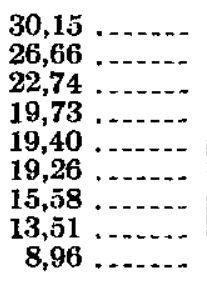 & $\begin{array}{l}0,680 \\
0,390 \\
0,230 \\
0,190 \\
0,140 \\
0,090 \\
0,045 \\
0,023 \\
-\ldots-.-\end{array}$ & $100^{\circ} \mathrm{C}$ & $\begin{array}{r}8 \\
16 \\
16 \\
24 \\
32 \\
40 \\
40 \\
48 \\
56 \\
64 \\
72 \\
7 \ldots\end{array}$ & 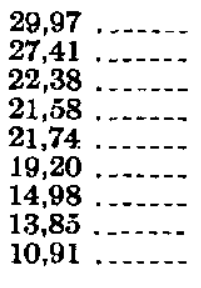 & $\begin{array}{l}2,300 \\
0,800 \\
0,450 \\
0,330 \\
0,280 \\
0,140 \\
0,023 \\
0,023 \\
\cdots\end{array}$ \\
\hline
\end{tabular}




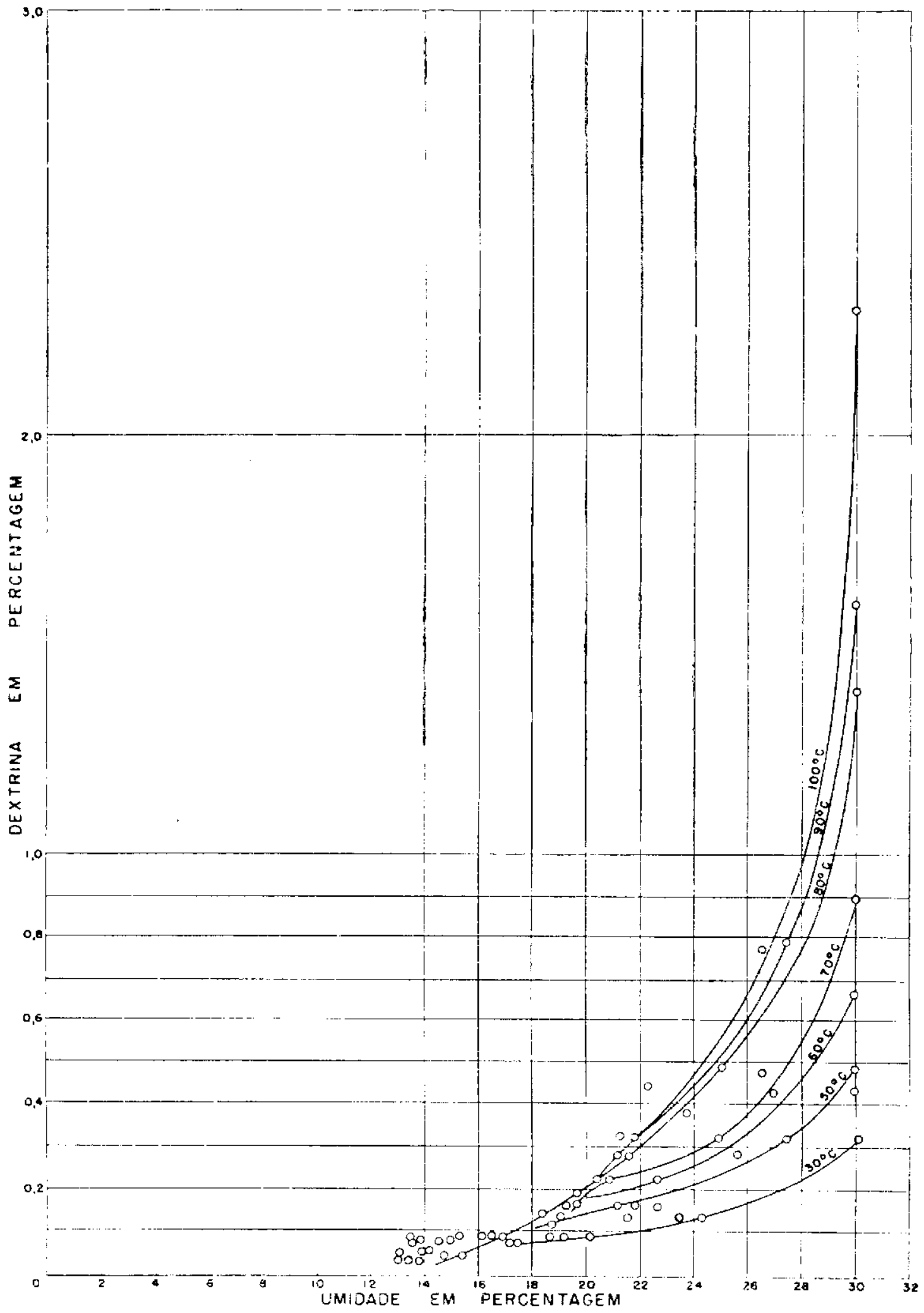

Figura 1.-Curvas das temperaturas de seeagem do amido, mostrando a correlaçāo entre umidade e a resultante percentagem da dextrina. A curva correspondente à secagem a $40^{\circ} \mathrm{C}$ nãa foi traçada, pelo fato de um dos valores ser muito discrepante. 


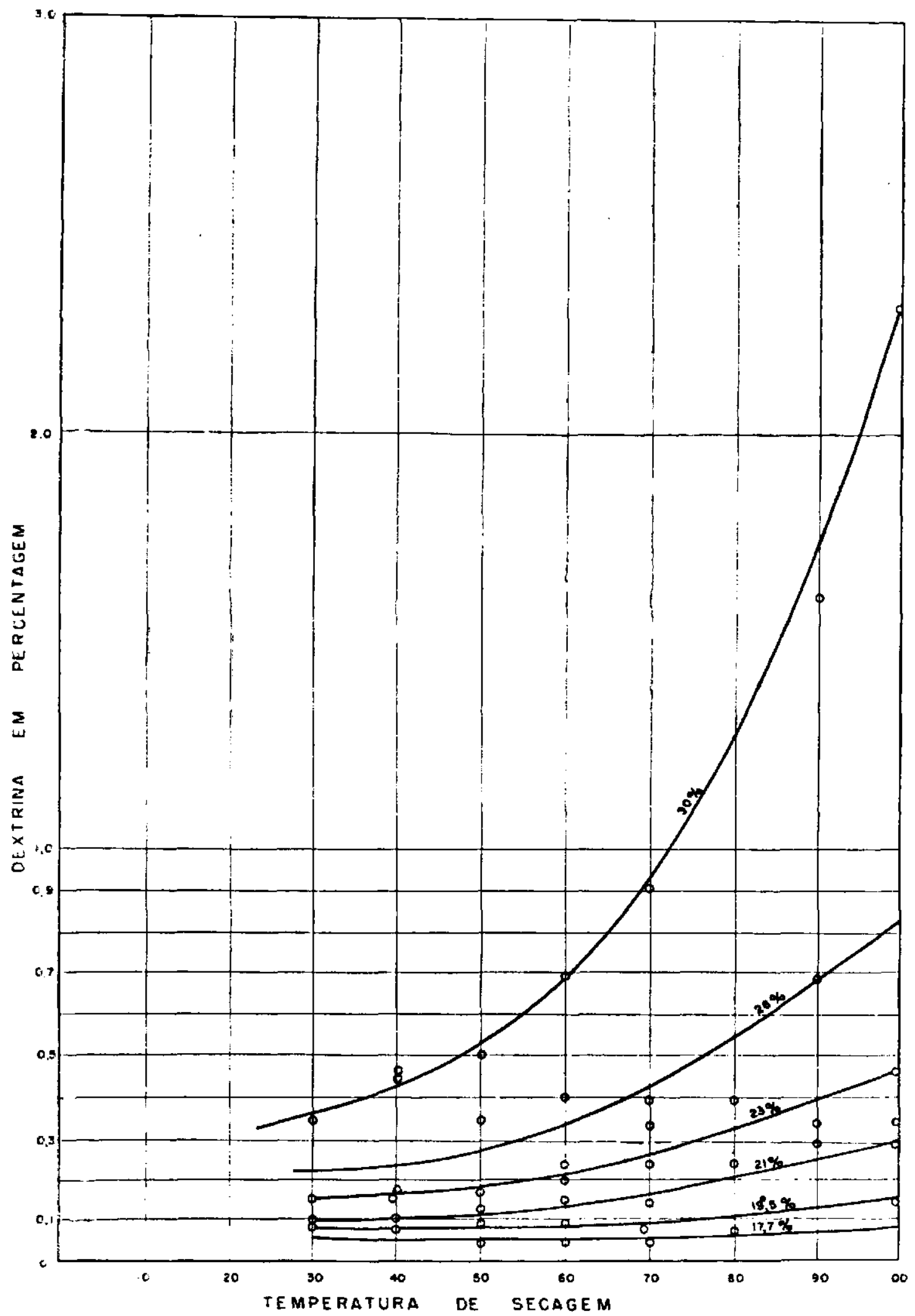

Fiavra 2.-Curvas das percentagens de umidades do amido mostrando a correlação entre a temperatura de secagem e a percentagem de dextrina. 
Com os dados obtidos, foram construídos os gráficos constantes das figuras 1,2 e 3 .

Pelo gráfico da figura 1 pode-se verificar a correlação existente entre a umidade inicial contida no amido e a percentagem de dextrina em secagens a diferentes temperaturas. Verifica-se que, quanto maior é a temperatura de secagem e quanto maior a umidade inicial do produto, maior é a percentagem de dextrina.

O gráfico da figura 2 evidencia que o amido, tendo $30 \%$ de umidade inicial, sêco à temperatura de 30 a $100^{\circ} \mathrm{C}$, terá sua formação de dextrinat aumentada de $0,3 \%$ a mais de $2 \%$, enquanto o mesmo amido, com $17,7 \%$ de umidade, mesmo sêco a $100^{\circ} \mathrm{C}$, não sofre pràticamente formação dessa substância.

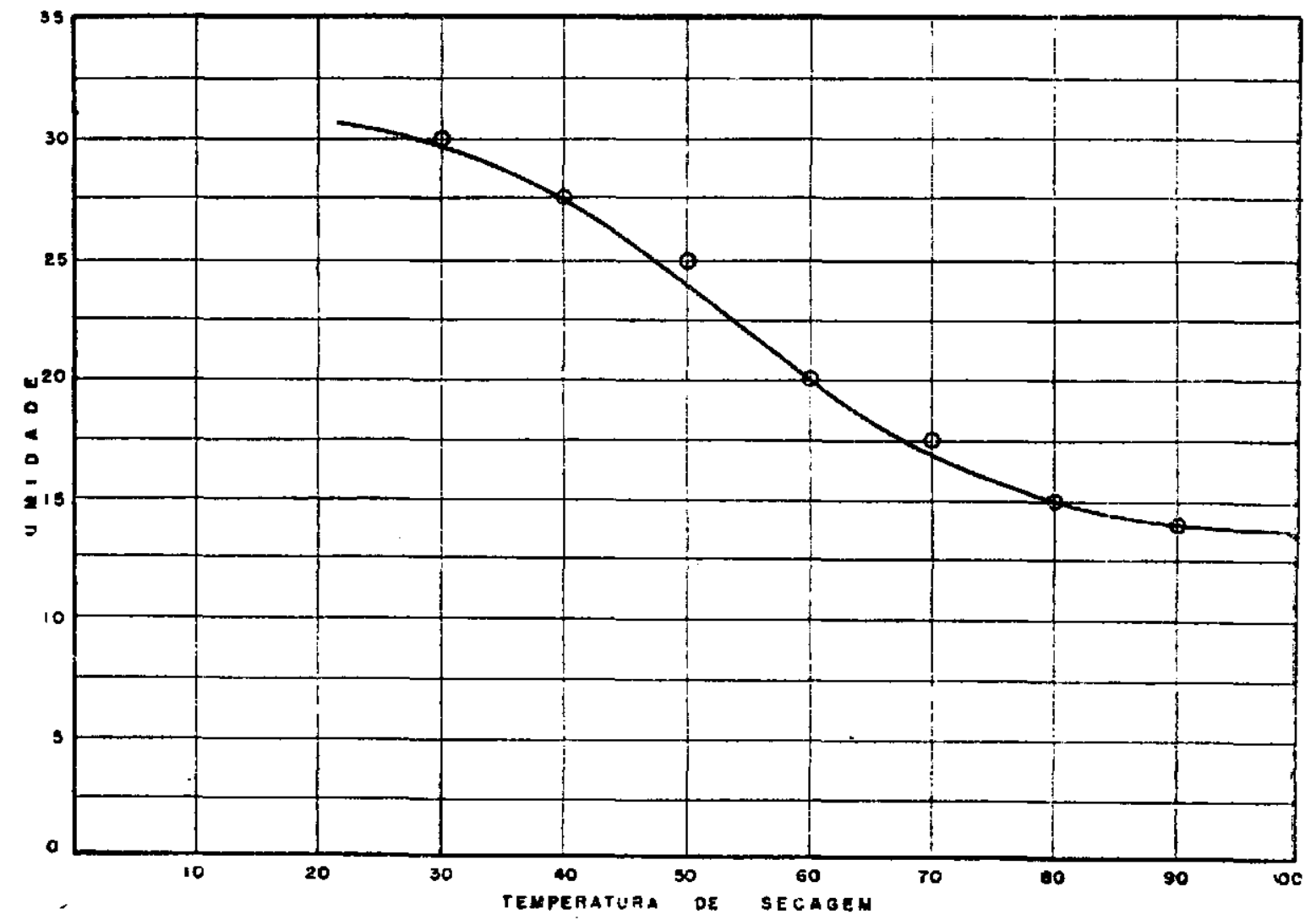

Figura 3.-Curra ideal de secagem do amido em secador a corrente contríria, para. que a percentagem de dextrinização esteja sempre abaixo de $0,3 \%$.

O gráfico da figura 3 mostra qual deve ser a marcha da secagem do amido com uma umidade inicial de $30 \%$, num secador em forma de túnel a "correntes contrárias", para que a percentagem de dextrina esteja sempre abaixo de $0,3 \%$. Uma boa secagem deverá ser feita sempre abaixo dia linha indicada neste gráfico.

Assim, o mínimo de formação de dextrina no produto é obtido quando as coordenadas da temperatura de secagem e umidade do produto deter- 
minam um ponto situado abaixo da referida curva. Exemplificando, tem-se : se o produto sair da centrífuga com $30 \%$ de umidade, êle deverá ser sêco à temperatura de $30^{\circ} \mathrm{C}$, e, à medida que êle vai secando, a temperatura pode ir aumentando até que, com $15 \%$ de umidade, êle poderá sofrer temperatura de $80^{\circ} \mathrm{C}$.

\section{3 - CONCLUSÕES}

Os resultados das experiências sôbre a secagem do amido pelo ar quente indicam que esta operação pode ser praticada com êxito, quando realizadi racionalmente. Verificou-se que os seguintes pontos são de importância para isso :

a) A umidade inicial do amido a secar deve ser a menor possível, não devendo exceder a $30 \%$, o que se obtém com uma boa centrifugação.

b) O secador deve ser baseado no princípio da contracorrente.

c) A marcha da secagem deve ser feita de modo que o amido mais sêco entre em contacto com ar mais quente, de acôrdo com as indicações de umidade e temperatura, expostas na figura 3.

\section{SUMMARY}

In the industrial production of starch from cassava roots (Manihot utilissima Pohi) in the State of São Paulo, the drying operation is carried out by the slow and costly method of vacuum dehydration. The need for a quicker and less expensive method led the writers to investigate the dehydration of raw starch by hot air. Studies were then made to determine the effect of water content of raw starch and temperature of hot air used in drying the starch on the amount of dextrins present in the deshydrated product.

Samples of raw starch with a water content ranging from 8 to 30 per cent were prepared by slow drying at $30^{\circ} \mathrm{C}$. The samples were arranged in comparable groups, including the whole range in water content, and each group was further dried by hot air at tem peratures from 30 to $100^{\circ} \mathrm{C}$. After the dehydration was completed, each sample was tested for dextrins.

The results of these tests indicated that hot air dehydration of raw, centrifuged starch is feasible, and that the degree of dextrin formation may be held below 0.3 per cent if the following procedure is followed:

a) the water content of the raw starch should be lowered to 30 per cent or preferably less by centrifugation ;

b) the hot air dehydrator should be of the "reversed current" type ;

c) the temperature of the air and rate of drying should be regulated according to the indications given in figure 3. 